A. ABKOWICZ and C. BREZINSKI (Lille)

\title{
ACCELERATION PROPERTIES OF THE HYBRID PROCEDURE FOR SOLVING LINEAR SYSTEMS
}

Abstract. The aim of this paper is to discuss the acceleration properties of the hybrid procedure for solving a system of linear equations. These properties are studied in a general case and in two particular cases which are illustrated by numerical examples.

1. The hybrid procedure. Let us consider the system of linear equations

$$
A x=b,
$$

where $A \in \mathbb{R}^{m \times m}$ and $x, b \in \mathbb{R}^{m}$. We denote by $\widetilde{x}$ the solution of (1).

Let $G=Z^{T} Z$ be a symmetric positive definite matrix. The $G$-inner product and the corresponding $G$-norm are respectively defined by $(x, y)_{G}=$ $(x, G y)$ and $\|x\|_{G}=\sqrt{(x, x)_{G}}$. The corresponding $G$-matrix norm is given by

$$
\|A\|_{G}=\sup _{x \neq 0} \frac{\|A x\|_{G}}{\|x\|_{G}}=\sqrt{\varrho\left(\left(Z A Z^{-1}\right)^{T} Z A Z^{-1}\right)} .
$$

We shall also use the notation $x \perp_{G} y$ if $(x, y)_{G}=0$. For simplicity, the subscript $G$ will be suppressed when unnecessary.

Let us now assume that two iterative methods for solving the system (1) are used simultaneously. Their iterates are denoted respectively by $x_{n}^{\prime}$ and $x_{n}^{\prime \prime}$ and the corresponding residual vectors by $r_{n}^{\prime}=b-A x_{n}^{\prime}$ and $r_{n}^{\prime \prime}=b-A x_{n}^{\prime \prime}$.

The hybrid procedure defined in [1] consists of constructing a new iterate $x_{n}$ and a new residual $r_{n}=b-A x_{n}$ by

$$
x_{n}=\alpha_{n} x_{n}^{\prime}+\left(1-\alpha_{n}\right) x_{n}^{\prime \prime}, \quad r_{n}=\alpha_{n} r_{n}^{\prime}+\left(1-\alpha_{n}\right) r_{n}^{\prime \prime},
$$

1991 Mathematics Subject Classification: 65F10, 65B05.

Key words and phrases: acceleration, linear equations, iterative methods. 
with

From the definition of $r_{n}$, we see that

$$
\alpha_{n}=-\frac{\left(r_{n}^{\prime}-r_{n}^{\prime \prime}, r_{n}^{\prime \prime}\right)}{\left(r_{n}^{\prime}-r_{n}^{\prime \prime}, r_{n}^{\prime}-r_{n}^{\prime \prime}\right)} .
$$

$$
\left\|r_{n}\right\|=\min _{\alpha}\left\|\alpha r_{n}^{\prime}+(1-\alpha) r_{n}^{\prime \prime}\right\| .
$$

We have

$$
\left(r_{n}, r_{n}^{\prime}\right)=\left(r_{n}, r_{n}^{\prime \prime}\right)=\left(r_{n}, r_{n}\right)
$$

and, setting $p_{n}=r_{n}^{\prime}-r_{n}^{\prime \prime}$, (2) can be written as

$$
r_{n}=r_{n}^{\prime \prime}-\frac{\left(p_{n}, r_{n}^{\prime \prime}\right)}{\left(p_{n}, p_{n}\right)} p_{n}, \quad r_{n}=r_{n}^{\prime}-\frac{\left(p_{n}, r_{n}^{\prime}\right)}{\left(p_{n}, p_{n}\right)} p_{n} .
$$

It is easy to check that

$$
\begin{aligned}
\left(r_{n}, r_{n}\right) & =\frac{\left(r_{n}^{\prime}, r_{n}^{\prime}\right)\left(r_{n}^{\prime \prime}, r_{n}^{\prime \prime}\right)-\left(r_{n}^{\prime}, r_{n}^{\prime \prime}\right)^{2}}{\left(r_{n}^{\prime}-r_{n}^{\prime \prime}, r_{n}^{\prime}-r_{n}^{\prime \prime}\right)} \\
& =\left(r_{n}^{\prime \prime}, r_{n}^{\prime \prime}\right)-\frac{\left(p_{n}, r_{n}^{\prime \prime}\right)^{2}}{\left(p_{n}, p_{n}\right)} \\
& =\left(r_{n}^{\prime}, r_{n}^{\prime}\right)-\frac{\left(p_{n}, r_{n}^{\prime}\right)^{2}}{\left(p_{n}, p_{n}\right)} .
\end{aligned}
$$

2. Properties of the hybrid procedure. We now study the acceleration properties of the hybrid procedure.

2.1. Asymptotic behavior of the hybrid procedure. Let $\theta_{n}$ be the angle between $Z r_{n}^{\prime}$ and $Z r_{n}^{\prime \prime}$. Using the relation $\left(r_{n}^{\prime}, r_{n}^{\prime \prime}\right)=\left\|r_{n}^{\prime}\right\|\left\|r_{n}^{\prime \prime}\right\| \cos \theta_{n}$ we have

and

$$
\alpha_{n}=-\frac{\left\|r_{n}^{\prime}\right\|\left\|r_{n}^{\prime \prime}\right\| \cos \theta_{n}-\left\|r_{n}^{\prime \prime}\right\|^{2}}{\left\|r_{n}^{\prime}\right\|^{2}-2\left\|r_{n}^{\prime}\right\|\left\|r_{n}^{\prime \prime}\right\| \cos \theta_{n}+\left\|r_{n}^{\prime \prime}\right\|^{2}}
$$

$$
\left\|r_{n}\right\|^{2}=\frac{\left\|r_{n}^{\prime}\right\|^{2}\left\|r_{n}^{\prime \prime}\right\|^{2}\left(1-\cos ^{2} \theta_{n}\right)}{\left\|r_{n}^{\prime}\right\|^{2}-2\left\|r_{n}^{\prime}\right\|\left\|r_{n}^{\prime \prime}\right\| \cos \theta_{n}+\left\|r_{n}^{\prime \prime}\right\|^{2}} .
$$

Setting $\varrho_{n}=\left\|r_{n}^{\prime}\right\| /\left\|r_{n}^{\prime \prime}\right\|$ we obtain

$$
\begin{aligned}
\alpha_{n} & =-\frac{\varrho_{n} \cos \theta_{n}-1}{\varrho_{n}^{2}-2 \varrho_{n} \cos \theta_{n}+1}, \\
\frac{\left\|r_{n}\right\|^{2}}{\left\|r_{n}^{\prime}\right\|^{2}} & =\frac{1-\cos ^{2} \theta_{n}}{\varrho_{n}^{2}-2 \varrho_{n} \cos \theta_{n}+1} \\
& =1-\frac{\left(\varrho_{n}-\cos \theta_{n}\right)^{2}}{\left(\varrho_{n}-\cos \theta_{n}\right)^{2}+\sin ^{2} \theta_{n}} \\
& =\frac{\sin ^{2} \theta_{n}}{\left(\varrho_{n}-\cos \theta_{n}\right)^{2}+\sin ^{2} \theta_{n}}
\end{aligned}
$$




$$
=\frac{\sin ^{2} \theta_{n}}{\varrho_{n}^{2}-2 \varrho_{n} \cos \theta_{n}+1}
$$

From these relations, we immediately obtain

Theorem 2.1. Suppose that the limit $\lim _{n \rightarrow \infty} \theta_{n}=\theta$ exists.

1. If $\lim _{n \rightarrow \infty} \varrho_{n}=0$ then $\lim _{n \rightarrow \infty} \alpha_{n}=1$.

2. If $\lim _{n \rightarrow \infty} \varrho_{n}=1$ and $\theta \neq 0$, then $\lim _{n \rightarrow \infty} \alpha_{n}=1 / 2$.

3. If $\lim _{n \rightarrow \infty} \varrho_{n}=\infty$ then $\lim _{n \rightarrow \infty} \alpha_{n}=0$.

This theorem shows that the hybrid procedure asymptotically selects the best method among the two.

Let us now consider the convergence behavior of $\left\|r_{n}\right\| /\left\|r_{n}^{\prime}\right\|$. From (10), we immediately have

TheOREM 2.2. If the limits $\lim _{n \rightarrow \infty} \varrho_{n}=\varrho$ and $\lim _{n \rightarrow \infty} \theta_{n}=\theta$ exist and $\varrho^{2}-2 \varrho \cos \theta+1 \neq 0$, then

$$
\lim _{n \rightarrow \infty} \frac{\left\|r_{n}\right\|^{2}}{\left\|r_{n}^{\prime}\right\|^{2}}=\frac{\sin ^{2} \theta}{\varrho^{2}-2 \varrho \cos \theta+1} \leq 1 .
$$

Remark 1 . Obviously if $\varrho \leq 1$, we also have $\lim _{n \rightarrow \infty}\left\|r_{n}\right\|^{2} /\left\|r_{n}^{\prime \prime}\right\|^{2} \leq 1$. Thus $\lim _{n \rightarrow \infty}\left\|r_{n}\right\| / \min \left(\left\|r_{n}^{\prime}\right\|,\left\|r_{n}^{\prime \prime}\right\|\right)$ exists and is not greater than 1 .

Similar results can be obtained by considering the ratio $\left\|r_{n}\right\|^{2} /\left\|r_{n}^{\prime \prime}\right\|^{2}$.

It must also be noticed that $\left\|r_{n}\right\|^{2} /\left\|r_{n}^{\prime}\right\|^{2}$ tends to 1 if and only if $\varrho=$ $\cos \theta$. This result comes out directly from (8) and we also get

THEOREM 2.3. A necessary and sufficient condition for the existence of an $N$ such that

$$
0 \leq\left\|r_{n}\right\|^{2} /\left\|r_{n}^{\prime}\right\|^{2}<1 \quad \text { for all } n \geq N
$$

is that $\left(r_{n}^{\prime}-r_{n}^{\prime \prime}, r_{n}^{\prime}\right) \neq 0$ for all $n \geq N$.

P r o of. Suppose that $\left(r_{n}^{\prime}-r_{n}^{\prime \prime}, r_{n}^{\prime}\right) \neq 0$ for all $n \geq N$. Thus we have

$$
\varrho_{n}-\cos \theta_{n}=\frac{\left\|r_{n}^{\prime}\right\|}{\left\|r_{n}^{\prime \prime}\right\|}-\frac{\left(r_{n}^{\prime}, r_{n}^{\prime \prime}\right)}{\left\|r_{n}^{\prime}\right\|\left\|r_{n}^{\prime \prime}\right\|}=\frac{\left(r_{n}^{\prime}, r_{n}^{\prime}-r_{n}^{\prime \prime}\right)}{\left\|r_{n}^{\prime}\right\|\left\|r_{n}^{\prime \prime}\right\|} \neq 0
$$

and, from (8), it follows that $\left\|r_{n}\right\|^{2} /\left\|r_{n}^{\prime}\right\|^{2}<1$. The reverse implication is proved similarly.

Let us now study some cases where $\left(r_{n}\right)$ converges to zero faster than $\left(r_{n}^{\prime}\right)$ and $\left(r_{n}^{\prime \prime}\right)$. From $(9)$, we have

THEOREM 2.4. If there are $\varrho$ and $N$ such that $0 \leq \varrho_{n} \leq \varrho<1$ for all $n \geq N$, then a necessary and sufficient condition for

$$
\lim _{n \rightarrow \infty}\left\|r_{n}\right\| /\left\|r_{n}^{\prime}\right\|=0
$$

to hold is that $\left(\theta_{n}\right)$ tends to 0 or $\pi$. 
Proof. First let us prove the sufficiency. Suppose that $\left(\theta_{n}\right)$ tends to 0 or $\pi$. Thus, since $\varrho_{n} \leq \varrho<1$, from (9) we have $\lim _{n \rightarrow \infty}\left\|r_{n}\right\| /\left\|r_{n}^{\prime}\right\|=0$.

To prove the necessity, suppose that $\lim _{n \rightarrow \infty}\left\|r_{n}\right\| /\left\|r_{n}^{\prime}\right\|=0$. The condition $\varrho_{n} \leq \varrho<1$ implies that $\sin \theta_{n}$ tends to 0 , which ends the proof.

Remark 2. Since $\varrho_{n}<1$ we have $\left\|r_{n}^{\prime}\right\|<\left\|r_{n}^{\prime \prime}\right\|$ for all $n \geq N$ and so

$$
\lim _{n \rightarrow \infty}\left\|r_{n}\right\| / \min \left(\left\|r_{n}^{\prime}\right\|,\left\|r_{n}^{\prime \prime}\right\|\right)=0
$$

Let us now study the case where $\left(\varrho_{n}\right)$ tends to 1 . From (10), we first have

Theorem 2.5. If $\lim _{n \rightarrow \infty} \varrho_{n}=1$, then a sufficient condition for

$$
\lim _{n \rightarrow \infty}\left\|r_{n}\right\| /\left\|r_{n}^{\prime}\right\|=0
$$

to hold is that $\left(\theta_{n}\right)$ tends to $\pi$.

Remark 3. Since $\lim _{n \rightarrow \infty} \varrho_{n}=1$, it follows that

$$
\lim _{n \rightarrow \infty}\left\|r_{n}\right\| / \min \left(\left\|r_{n}^{\prime}\right\|,\left\|r_{n}^{\prime \prime}\right\|\right)=0
$$

Another result in the case where $\left(\varrho_{n}\right)$ tends to 1 is given by

THEOREM 2.6. If $\left\|r_{n}^{\prime}\right\| /\left\|r_{n}^{\prime \prime}\right\|=1+a_{n}$ with $\lim _{n \rightarrow \infty} a_{n}=0$, then $a$ sufficient condition for

$$
\lim _{n \rightarrow \infty}\left\|r_{n}\right\| /\left\|r_{n}^{\prime}\right\|=0
$$

to hold is that $\theta_{n}=o\left(a_{n}\right)$.

Proof. We have

$$
\cos \theta_{n}=1-\theta_{n}^{2} / 2+\mathcal{O}\left(\theta_{n}^{4}\right), \quad \sin \theta_{n}=\theta_{n}+\mathcal{O}\left(\theta_{n}^{3}\right) .
$$

Replacing in formula (10), we have

$$
\begin{aligned}
\frac{\left\|r_{n}\right\|}{\left\|r_{n}^{\prime}\right\|} & =\frac{\sin ^{2} \theta_{n}}{\varrho_{n}^{2}-2 \varrho_{n} \cos \theta_{n}+1} \\
& =\frac{\left(\theta_{n}\left(1+\mathcal{O}\left(\theta_{n}^{2}\right)\right)\right)^{2}}{\left(1+a_{n}\right)^{2}-2\left(1+a_{n}\right)\left(1-\theta_{n}^{2} / 2+\mathcal{O}\left(\theta_{n}^{4}\right)\right)+1} \\
& =\frac{\theta_{n}^{2}\left(1+\mathcal{O}\left(\theta_{n}^{2}\right)\right)}{a_{n}^{2}+\theta_{n}^{2}+a_{n} \theta_{n}^{2}+\left(1+a_{n}\right) \mathcal{O}\left(\theta_{n}^{4}\right)} \\
& =\frac{1+\mathcal{O}\left(\theta_{n}^{2}\right)}{\left(a_{n} / \theta_{n}\right)^{2}+1+a_{n}+\left(1+a_{n}\right) \mathcal{O}\left(\theta_{n}^{2}\right)}
\end{aligned}
$$

and the result follows.

Remark 4. Since $\lim _{n \rightarrow \infty} \varrho_{n}=1$, the conclusion of Remark 3 still holds. 
Another presentation consists in considering the angle $\vartheta_{n}$ between $Z r_{n}^{\prime}$ and $Z p_{n}$. From (6) we have $\left\|r_{n}\right\|^{2}=\left\|r_{n}^{\prime}\right\|^{2} \sin ^{2} \vartheta_{n}$. Directly from this equation we obtain

TheOREm 2.7. If there exists $\vartheta \neq \pi / 2$ such that $\lim _{n \rightarrow \infty} \vartheta_{n}=\vartheta$ then $\lim _{n \rightarrow \infty}\left\|r_{n}\right\| /\left\|r_{n}^{\prime}\right\|=|\sin \vartheta|<1$.

Also, we have

THEOREM 2.8. $\lim _{n \rightarrow \infty}\left\|r_{n}\right\| /\left\|r_{n}^{\prime}\right\|=0$ if and only if $\left(\vartheta_{n}\right)$ tends to 0 or $\pi$.

These results are simpler than the preceding ones, in particular those of Theorems 2.2-2.4.

Remark 5. Similarly, if we denote by $\varphi_{n}$ the angle between $Z r_{n}^{\prime \prime}$ and $Z p_{n}$, we have $\left\|r_{n}\right\|^{2}=\left\|r_{n}^{\prime \prime}\right\|^{2} \sin ^{2} \varphi_{n}$. Obviously $\theta_{n}=\varphi_{n}-\vartheta_{n}$.

2.2. Geometric behavior of the hybrid procedure. A sphere in $\mathbb{R}^{m}$ with respect to the $G$-norm will be denoted by

$$
\Upsilon_{G}(q, r)=\left\{y \in \mathbb{R}^{m}:\|y-q\|_{G}=r\right\} .
$$

We have the following properties:

Property 1. $r_{n} \in \Upsilon_{G}\left(r_{n}^{\prime} / 2,\left\|r_{n}^{\prime}\right\|_{G} / 2\right) \cap \Upsilon_{G}\left(r_{n}^{\prime \prime} / 2,\left\|r_{n}^{\prime \prime}\right\|_{G} / 2\right)$.

Proof. By definition, we have $\left(r_{n}, r_{n}\right)=\left(r_{n}, r_{n}^{\prime}\right)=\left(r_{n}, r_{n}^{\prime \prime}\right)$. Computing $\left\|r_{n}-r_{n}^{\prime} / 2\right\|^{2}$ we get

$$
\left\|r_{n}-r_{n}^{\prime} / 2\right\|^{2}=\left\|r_{n}\right\|^{2}-\left(r_{n}, r_{n}^{\prime}\right)+\frac{1}{4}\left\|r_{n}^{\prime}\right\|^{2}=\frac{1}{4}\left\|r_{n}^{\prime}\right\|^{2} .
$$

In the same way, we can prove that $\left\|r_{n}-r_{n}^{\prime \prime} / 2\right\|^{2}=\frac{1}{4}\left\|r_{n}^{\prime \prime}\right\|^{2}$ and the result follows.

Let us denote by $e_{n}=\widetilde{x}-x_{n}, e_{n}^{\prime}=\widetilde{x}-x_{n}^{\prime}, e_{n}^{\prime \prime}=\widetilde{x}-x_{n}^{\prime \prime}$ the error vectors corresponding respectively to $x_{n}, x_{n}^{\prime}, x_{n}^{\prime \prime}$. Using the relation $r_{n}=A e_{n}$ and the preceding property we have

PROPERTY 2.

$$
e_{n} \in \Upsilon_{A^{T} G A}\left(e_{n}^{\prime} / 2,\left\|e_{n}^{\prime}\right\|_{A^{T} G A} / 2\right) \cap \Upsilon_{A^{T} G A}\left(e_{n}^{\prime \prime} / 2,\left\|e_{n}^{\prime \prime}\right\|_{A^{T} G A} / 2\right) .
$$

The hybrid procedure is a projection method because there exists a matrix $\wp_{n} \in \mathbb{R}^{m \times m}$ such that

$$
r_{n}=\wp_{n} r_{n}^{\prime}=\wp_{n} r_{n}^{\prime \prime} \quad \text { with } \wp_{n}=I-\frac{p_{n} p_{n}^{T} G}{p_{n}^{T} G p_{n}} .
$$

It is easy to see that $\wp_{n}^{2}=\wp_{n}$ and $\left(G \wp_{n}\right)^{T}=G \wp_{n}$. So $\wp_{n}$ is a $G$-orthogonal projection. By definition of $\wp_{n}$ we get

$$
\begin{array}{ll}
\wp_{n} v=v & \text { if } v \perp_{G} p_{n}, \\
\wp_{n} v=0 & \text { if } v \in \operatorname{span}\left\{p_{n}\right\} .
\end{array}
$$


The above results can be considered as a generalization of the results given in $[8]$.

3. Applications. It seems quite difficult to obtain more theoretical results on the convergence of the hybrid procedure in the general case. So, $\left(r_{n}^{\prime}\right)$ being an arbitrary sequence of residual vectors, we shall assume that we are in one of the following particular cases:

(i) $r_{n}^{\prime \prime}=B r_{n-1}$,

(ii) $r_{n}^{\prime \prime}=B r_{n}^{\prime}$.

Such a situation arises, for example, if we consider a splitting of the matrix $A$,

$$
A=M-N,
$$

and if $x_{n}^{\prime \prime}$ is obtained from $y$ (equal to $x_{n-1}$ or $x_{n}^{\prime}$ ) by

$$
x_{n}^{\prime \prime}=M^{-1} N y+M^{-1} b .
$$

In this case the associated residual has the form

$$
\begin{aligned}
r_{n}^{\prime \prime} & =b-A x_{n}^{\prime \prime}=b-A\left(M^{-1} N y+M^{-1} b\right) \\
& =b-(M-N)\left(M^{-1} N y+M^{-1} b\right)=N M^{-1}(b-A y) .
\end{aligned}
$$

Thus we have $B=N M^{-1}$ with $y=x_{n-1}$ (case (i)) and $y=x_{n-1}^{\prime}$ (case (ii)). It must be noticed that $B=I-A M^{-1}$. This situation also holds if $B=$ $I-A C$ with $C$ an arbitrary matrix. In this case, we have

$$
x_{n}=\alpha_{n} x_{n}^{\prime}+\left(1-\alpha_{n}\right)(y+C(b-A y)) .
$$

(We are indebted to one of the referees for this remark.)

3.1. Case (i). Let $r_{n}$ be computed by the hybrid procedure from $r_{n}^{\prime \prime}=$ $B r_{n-1}$ and $r_{n}^{\prime}$. We have

$$
r_{n}=\alpha_{n} r_{n}^{\prime}+\left(1-\alpha_{n}\right) B r_{n-1}
$$

and we get

LEMma 3.1. Let $r_{0}=r_{0}^{\prime}$. Then, for all $n \geq 1$,

$H 1(n)$

$$
r_{n}=\sum_{i=0}^{n} a_{i}^{(n)} B^{n-i} r_{i}^{\prime}
$$

with

$H 2(n)$

$$
\sum_{i=0}^{n} a_{i}^{(n)}=1 .
$$

Proof. $a_{0}^{(0)}=1$ and so $H 1(0)$ and $H 2(0)$ are true. Suppose that $H 1(n-1)$ and $H 2(n-1)$ hold. From the definition of $r_{n}$ and from $H 1(n-1)$, 
we get

$$
\begin{aligned}
r_{n} & =\alpha_{n} r_{n}^{\prime}+\left(1-\alpha_{n}\right) B r_{n-1}=\alpha_{n} r_{n}^{\prime}+\left(1-\alpha_{n}\right) B \sum_{i=0}^{n-1} a_{i}^{(n-1)} B^{n-1-i} r_{i}^{\prime} \\
& =\alpha_{n} r_{n}^{\prime}+\sum_{i=0}^{n-1}\left(1-\alpha_{n}\right) a_{i}^{(n-1)} B^{n-i} r_{i}^{\prime}=\sum_{i=0}^{n} a_{i}^{(n)} B^{n-i} r_{i}^{\prime},
\end{aligned}
$$

where the $a_{i}^{(n)}$ 's are given by

$$
\begin{aligned}
& a_{i}^{(n)}=\left(1-\alpha_{n}\right) a_{i}^{(n-1)}, \quad i=0, \ldots, n-1, \\
& a_{n}^{(n)}=\alpha_{n} .
\end{aligned}
$$

Thus $H 1(n)$ is true with $H 2(n)$ obviously satisfied.

R e mark 6 . When $r_{n}^{\prime}$ is computed by a polynomial method of the form $r_{n}^{\prime}=P_{n}(B) r_{0}^{\prime}$ then $r_{n}=Q_{n}(B) r_{0}^{\prime}$ with $Q_{n}$ given by $Q_{n}(t)=\alpha_{n} P_{n}(t)+$ $\left(1-\alpha_{n}\right) t Q_{n-1}(t)$.

Let us now prove other results. We have

TheOREm 3.2. Let $\gamma$ be an eigenvector of B. If $r_{n}=c_{n} \gamma+a_{n}$ with

$$
\lim _{n \rightarrow \infty}\left(\gamma, r_{n}^{\prime}\right) /\left\|r_{n}^{\prime}\right\|=\|\gamma\| \text { and } \lim _{n \rightarrow \infty}\left\|a_{n}\right\| / c_{n}=0
$$

then $\lim _{n \rightarrow \infty}\left\|r_{n}\right\| /\left\|r_{n}^{\prime}\right\|=0$.

Proof. Let $\theta_{n}$ be the angle between $Z B r_{n-1}$ and $Z r_{n}^{\prime}$. We have

$$
\begin{aligned}
\left(B r_{n-1}, r_{n}^{\prime}\right)^{2} & =c_{n-1}^{2} \lambda^{2}\left(\gamma, r_{n}^{\prime}\right)^{2}+\left(B a_{n-1}, r_{n}^{\prime}\right)^{2}+2 c_{n-1} \lambda\left(\gamma, r_{n}^{\prime}\right)\left(B a_{n-1}, r_{n}^{\prime}\right), \\
\left\|B r_{n-1}\right\|^{2} & =c_{n-1}^{2} \lambda^{2}\|\gamma\|^{2}+\left\|B a_{n-1}\right\|^{2}+2 c_{n-1} \lambda\left(\gamma, B a_{n-1}\right),
\end{aligned}
$$

where $\lambda$ is the eigenvalue of $B$ corresponding to $\gamma$. Thus

$$
\begin{aligned}
\lim _{n \rightarrow \infty} \cos ^{2} \theta_{n} & =\lim _{n \rightarrow \infty} \frac{\left(B r_{n-1}, r_{n}^{\prime}\right)^{2}}{\left\|B r_{n-1}\right\|^{2}\left\|r_{n}^{\prime}\right\|^{2}} \\
& =\lim _{n \rightarrow \infty}\left[\frac{\lambda^{2} \frac{\left(\gamma, r_{n}^{\prime}\right)^{2}}{\left\|r_{n}^{\prime}\right\|^{2}}+\frac{\left(B a_{n-1}, r_{n}^{\prime}\right)^{2}}{c_{n-1}^{2}\left\|r_{n}^{\prime}\right\|^{2}}+2 \lambda \frac{\left(\gamma, r_{n}^{\prime}\right)\left(B a_{n-1}, r_{n}^{\prime}\right)}{c_{n-1}\left\|r_{n}^{\prime}\right\|^{2}}}{\lambda^{2}\|\gamma\|^{2}+\frac{\left\|B a_{n-1}\right\|^{2}}{c_{n-1}^{2}}+2 \lambda \frac{\left(\gamma, a_{n-1}\right)}{c_{n-1}}}\right] \\
& =1
\end{aligned}
$$

and the result follows from Theorem 2.4.

From the minimization property of $r_{n}$ we have

$$
\left\|r_{n}\right\| \leq\left\|B r_{n-1}\right\| \leq\|B\|\left\|r_{n-1}\right\|
$$

and thus $\left\|r_{n}\right\| \leq\left\|r_{n-1}\right\|$ if $\|B\| \leq 1$. 
In particular, consider a splitting $A=M-N$ of the matrix $A$. Premultiplying the system (1) from the right by $M^{-1}$ we get a new system of the form

with

$$
A^{(M)} x=b^{(M)}
$$

$$
B^{(M)}=M^{-1} N, \quad A^{(M)}=I-B^{(M)}, \quad b^{(M)}=M^{-1} b .
$$

Applying the method described above to this new system, we get

$$
\left\|r_{n}\right\| \leq\left\|B^{(M)}\right\|\left\|r_{n-1}\right\| \text {. }
$$

Thus, a good choice of $B^{(M)}$ is equivalent to a good choice of the preconditioner $M$ from the right-hand side.

When $B=I$, the method is called the Minimal Residual Smoothing (MRS) algorithm. It was introduced in $[6,7]$ and applied to some well known methods. For more details, see [2, 9-12].

We now apply it to an error-minimization method [4]. Set $e_{n}^{\prime}=\widetilde{x}-x_{n}^{\prime}$ and $e_{n}=\widetilde{x}-x_{n}$. Let $\varphi$ be any norm in $\mathbb{R}^{m}$. For any $x \in \mathbb{R}^{m}$ we denote by $z(x)$ a vector such that

$$
(z(x), x)=\varphi(x) .
$$

This is called a decomposition of the norm $\varphi$. Such decompositions were introduced by Gastinel [3] for the case of the $l_{1}$-norm.

Let $x_{0}^{\prime}$ be a given vector. The Transformed Norm Decomposition Method (TNDE) [4] is defined by

$$
r_{0}^{\prime}=b-A x_{0}^{\prime}, \quad p_{0}^{\prime}=A^{T} z_{0},
$$

and for $n=0,1, \ldots$,

$$
\begin{aligned}
& x_{n+1}^{\prime}=x_{n}^{\prime}-\beta_{n} p_{n}^{\prime}, \quad r_{n+1}^{\prime}=r_{n}^{\prime}+\beta_{n} A p_{n}^{\prime}, \\
& p_{n+1}^{\prime}=A^{T} z_{n+1}+\sum_{i=0}^{n} \gamma_{n+1}^{(i)} p_{i}^{\prime},
\end{aligned}
$$

where $z_{i}$ is such that $\left(z_{i}, r_{i}\right)=\varphi\left(r_{i}\right)$. The coefficients $\beta_{n}$ and $\gamma_{n+1}^{(i)}$ are given by

and

$$
\beta_{n}=\frac{\left(p_{n}^{\prime}, e_{n}^{\prime}\right)}{\left(p_{n}^{\prime}, p_{n}^{\prime}\right)}=-\frac{\varphi\left(r_{n}^{\prime}\right)}{\left(p_{n}^{\prime}, p_{n}^{\prime}\right)}
$$

$$
\gamma_{n+1}^{(i)}=-\frac{\left(p_{i}^{\prime}, A^{T} z_{n+1}\right)}{\left(p_{i}^{\prime}, p_{i}^{\prime}\right)}, \quad i=0, \ldots, n .
$$

The sequence $\left(e_{n}^{\prime}=\widetilde{x}-x_{n}^{\prime}\right)$ has the following properties:

1. $e_{n}^{\prime} \in V_{n}=e_{0}^{\prime}+\operatorname{span}\left\{p_{0}^{\prime}, \ldots, p_{n}^{\prime}\right\}$,

2. $V_{n-1} \subset V_{n}$,

3. $\left\|e_{n}^{\prime}\right\|=\min _{e \in V_{n}}\|e\|$,

4. $\left\|e_{n}^{\prime}\right\| \leq\left\|e_{n-1}^{\prime}\right\|$. 
If the MRS is applied to the TNDE, that is, to the sequence $\left(r_{n}^{\prime}\right)$ defined above with $r_{0}=r_{0}^{\prime}$, then we have

Theorem 3.3. If $\left.\alpha_{n} \in\right] 0,1\left[\right.$ then $\left\|e_{n}\right\| \leq\left\|e_{n-1}\right\|$.

Proof. We have

$$
e_{n-1}=\alpha_{n-1} e_{n-1}^{\prime}+\left(1-\alpha_{n-1}\right) e_{n-2} .
$$

It follows that $e_{n-1}=\sum_{i=1}^{n-1} a_{i}^{(n-1)} e_{i}^{\prime} \in V_{n-1}$ for all $n$. Thus, from property 3 ,

$$
\left\|e_{n-1}^{\prime}\right\| \leq\left\|e_{n-1}\right\|
$$

Using property 4 , we get

$$
\begin{aligned}
\left\|e_{n}\right\| & \leq \alpha_{n}\left\|e_{n}^{\prime}\right\|+\left(1-\alpha_{n}\right)\left\|e_{n-1}\right\| \leq \alpha_{n}\left\|e_{n-1}^{\prime}\right\|+\left(1-\alpha_{n}\right)\left\|e_{n-1}\right\| \\
& \leq \alpha_{n}\left\|e_{n-1}\right\|+\left(1-\alpha_{n}\right)\left\|e_{n-1}\right\|=\left\|e_{n-1}\right\|
\end{aligned}
$$

and the result follows.

3.2. Case (ii). Suppose now that $r_{n}$ is given by

$$
r_{n}=\alpha_{n} r_{n}^{\prime}+\left(1-\alpha_{n}\right) B r_{n}^{\prime}
$$

with $B=I-A M^{-1}$. Then $r_{n}$ can be written as

$$
r_{n}=r_{n}^{\prime}-\frac{\left(A M^{-1} r_{n}^{\prime}, r_{n}^{\prime}\right)}{\left(A M^{-1} r_{n}^{\prime}, A M^{-1} r_{n}^{\prime}\right)} A M^{-1} r_{n}^{\prime}
$$

Remark 7. If $r_{n}^{\prime}=r_{n-1}$ and $M=I$, then the hybrid procedure is identical to the Minimal Residual Method.

Definition 1. Consider two vector sequences $\left(u_{n}\right),\left(v_{n}\right) \in \mathbb{R}^{m}$ such that $\lim _{n \rightarrow \infty} u_{n}=u$ and $\lim _{n \rightarrow \infty} v_{n}=v$. We say that $\left(u_{n}\right)$ converges with the same speed as $\left(v_{n}\right)$ if there exists $N$ such that for all $n \geq N$ there are $M_{n} \in \mathbb{R}^{m \times m}$ and $a_{n} \in \mathbb{R}^{m}$ with $\left\|a_{n}\right\| \leq \varepsilon$ such that

- $v_{n+1}=M_{n} v_{n}$,

- $u_{n+1}=M_{n} u_{n}+a_{n}$.

Lemma 3.4. Suppose that there exists $N$ such that for all $n \geq N$, there is $M_{n} \in \mathbb{R}^{m \times m}$ such that $r_{n+1}^{\prime}=M_{n} r_{n}^{\prime}$ and $A M^{-1} M_{n}=M_{n} A M^{-1}$. If $\lim _{n \rightarrow \infty} \alpha_{n}=\alpha$ exists and if there is $K$ such that $\left\|M_{n}\right\|<K$ for all $n$, then $\left(r_{n}\right)$ converges with the same speed as $\left(r_{n}^{\prime}\right)$.

Proof. If $\left(\alpha_{n}\right)$ converges, then there is a sequence $\left(\varepsilon_{n}\right)$ with $\lim _{n \rightarrow \infty} \varepsilon_{n}$ $=0$ such that $\alpha_{n+1}=\alpha_{n}-\varepsilon_{n}$ for all $n$. Setting $a_{n}=\varepsilon_{n} A M^{-1} M_{n} r_{n}^{\prime}$, we get from the definition

$$
\begin{aligned}
r_{n+1} & =r_{n+1}^{\prime}-\left(1-\alpha_{n+1}\right) A M^{-1} r_{n+1}^{\prime}=M_{n} r_{n}^{\prime}-\left(1-\alpha_{n}+\varepsilon_{n}\right) A M^{-1} M_{n} r_{n}^{\prime} \\
& =M_{n}\left(r_{n}^{\prime}-\left(1-\alpha_{n}\right) A M^{-1} r_{n}^{\prime}\right)+\varepsilon_{n} A M^{-1} M_{n} r_{n}^{\prime}=M_{n} r_{n}+a_{n} .
\end{aligned}
$$

Obviously $\lim _{n \rightarrow \infty} a_{n}=0$ and the result follows. 
We now assume that $r_{n}^{\prime}=c_{n} \gamma+a_{n}$, where $c_{n} \in \mathbb{R}, a_{n} \in \mathbb{R}^{m}$, and that $\gamma$ is an eigenvector of $B$. In this case we get

LEMmA 3.5. Let $\gamma$ be an eigenvector of $B$. If $r_{n}^{\prime}=c_{n} \gamma+a_{n}$, then there are $K \in \mathbb{R}$ and $M_{n} \in \mathbb{R}^{m}$ such that for all $n,\left\|M_{n}\right\| \leq K$ and $r_{n}=M_{n} a_{n}$.

Proof. We know that

$$
r_{n}=\wp_{n} r_{n}^{\prime}=c_{n} \wp_{n} \gamma+\wp_{n} a_{n}
$$

with

$$
\wp_{n}=I-p_{n} p_{n}^{T} G /\left(p_{n}^{T} G p_{n}\right),
$$

where $p_{n}=A M^{-1} r_{n}^{\prime}$. Premultiplying $p_{n}$ by $\wp_{n}$ we get

$$
0=\wp_{n} p_{n}=(1-\lambda) c_{n} \wp_{n} \gamma+\wp_{n} A M^{-1} a_{n},
$$

where $\lambda$ is the eigenvalue of $B$ corresponding to $\gamma$. Thus, since $A$ is assumed to be regular,

$$
c_{n} \wp_{n} \gamma=-\frac{1}{1-\lambda} \wp_{n} A M^{-1} a_{n}
$$

Setting

$$
M_{n}=\wp_{n}\left(I-\frac{1}{1-\lambda} A M^{-1}\right),
$$

we get $r_{n}=M_{n} a_{n}$. The matrix $\wp_{n}$ is a $G$-orthogonal projection and thus $\left\|\wp_{n}\right\|_{G}=1$. It follows that

$$
\left\|M_{n}\right\| \leq 1+\frac{1}{|1-\lambda|}\left\|A M^{-1}\right\|
$$

which ends the proof.

Re mark 8. As a consequence of Lemma 3.5 we have $\left\|r_{n}\right\|=\mathcal{O}\left(\left\|a_{n}\right\|\right)$.

From Theorem 2.8, we easily get

THEOREM 3.6. Let $\gamma$ be an eigenvector of $B$ with the corresponding eigenvalue $\lambda$. If $r_{n}^{\prime}=c_{n} \gamma+a_{n}$ with $\lim _{n \rightarrow \infty}\left\|a_{n}\right\| / c_{n}=0$ then

$$
\lim _{n \rightarrow \infty} \alpha_{n}=-\frac{\lambda}{1-\lambda} \quad \text { and } \quad \lim _{n \rightarrow \infty} \frac{\left\|r_{n}\right\|}{\left\|r_{n}^{\prime}\right\|}=0
$$

Proof. We have

$r_{n}^{\prime}=c_{n} \gamma+a_{n}, \quad B r_{n}^{\prime}=\lambda c_{n} \gamma+B a_{n}, \quad A M^{-1} r_{n}^{\prime}=(1-\lambda) c_{n} \gamma+A M^{-1} a_{n}$, and 


$$
\begin{aligned}
\lim _{n \rightarrow \infty} \alpha_{n}= & -\frac{\left(B r_{n}^{\prime}, A M^{-1} r_{n}^{\prime}\right)}{\left(A M^{-1} r_{n}^{\prime}, A M^{-1} r_{n}^{\prime}\right)} \\
= & \lim _{n \rightarrow \infty}\left[-\frac{\lambda(1-\lambda) c_{n}^{2}(\gamma, \gamma)+c_{n} \lambda\left(\gamma, A M^{-1} a_{n}\right)}{(1-\lambda)^{2} c_{n}^{2}(\gamma, \gamma)+2(1-\lambda) c_{n}\left(\gamma, A M^{-1} a_{n}\right)+\left(A M^{-1} a_{n}, A M^{-1} a_{n}\right)}\right. \\
& \left.+\frac{(1-\lambda) c_{n}\left(B a_{n}, \gamma\right)+\left(B a_{n}, A M^{-1} a_{n}\right)}{(1-\lambda)^{2} c_{n}^{2}(\gamma, \gamma)+2(1-\lambda) c_{n}\left(\gamma, A M^{-1} a_{n}\right)+\left(A M^{-1} a_{n}, A M^{-1} a_{n}\right)}\right] \\
= & \lim _{n \rightarrow \infty}\left[-\frac{\lambda(1-\lambda)(\gamma, \gamma)+\lambda \frac{\left(\gamma, A M^{-1} a_{n}\right)}{c_{n}}}{(1-\lambda)^{2}(\gamma, \gamma)+2(1-\lambda) \frac{\left(\gamma, A M^{-1} a_{n}\right)}{c_{n}}+\frac{\left(A M^{-1} a_{n}, A M^{-1} a_{n}\right)}{c_{n}^{2}}}\right. \\
& \left.+\frac{(1-\lambda) \frac{\left(B a_{n}, \gamma\right)}{c_{n}}+\frac{\left(B a_{n}, A M^{-1} a_{n}\right)}{c_{n}^{2}}}{(1-\lambda)^{2}(\gamma, \gamma)+2(1-\lambda) \frac{\left(\gamma, A M^{-1} a_{n}\right)}{c_{n}}+\frac{\left(A M^{-1} a_{n}, A M^{-1} a_{n}\right)}{c_{n}^{2}}}\right] \\
= & -\frac{\lambda}{1-\lambda} .
\end{aligned}
$$

Let $\theta_{n}$ be the angle between $Z r_{n}^{\prime}$ and $Z A M^{-1} r_{n}^{\prime}$. Replacing $r_{n}^{\prime}$ and $A M^{-1} r_{n}^{\prime}$ by their expressions above, we also get

$$
\begin{aligned}
\lim _{n \rightarrow \infty} \cos ^{2} \theta_{n}= & \lim _{n \rightarrow \infty} \frac{\left(r_{n}^{\prime}, A M^{-1} r_{n}^{\prime}\right)^{2}}{\left\|r_{n}^{\prime}\right\|^{2}\left\|A M^{-1} r_{n}^{\prime}\right\|^{2}} \\
= & \lim _{n \rightarrow \infty}\left[\frac{1}{c_{n}^{2}(\gamma, \gamma)+2 c_{n}\left(\gamma, a_{n}\right)+\left(a_{n}, a_{n}\right)}\right. \\
& \left.\times \frac{\left[(1-\lambda) c_{n}^{2}(\gamma, \gamma)+c_{n}\left(\gamma, A M^{-1} a_{n}\right)+(1-\lambda) c_{n}\left(a_{n}, \gamma\right)+\left(a_{n}, A M^{-1} a_{n}\right)\right]^{2}}{(1-\lambda)^{2} c_{n}^{2}(\gamma, \gamma)+2(1-\lambda) c_{n}\left(\gamma, A M^{-1} a_{n}\right)+\left(A M^{-1} a_{n}, A M^{-1} a_{n}\right)}\right] \\
= & \lim _{n \rightarrow \infty}\left[\frac{1}{(\gamma, \gamma)+2 \frac{\left(\gamma, a_{n}\right)}{c_{n}}+\frac{\left(a_{n}, a_{n}\right)}{c_{n}^{2}}}\right. \\
& \left.\times \frac{\left[(1-\lambda)(\gamma, \gamma)+\frac{\left(\gamma, A M^{-1} a_{n}\right)}{c_{n}}+(1-\lambda) \frac{\left(a_{n}, \gamma\right)}{c_{n}}+\frac{\left(a_{n}, A M^{-1} a_{n}\right)}{c_{n}^{2}}\right]^{2}}{(1-\lambda)^{2}(\gamma, \gamma)+2(1-\lambda) \frac{\left(\gamma, A M^{-1} a_{n}\right)}{c_{n}}+\frac{\left(A M^{-1} a_{n}, A M^{-1} a_{n}\right)}{c_{n}^{2}}}\right] \\
= & 1
\end{aligned}
$$

and the result follows by Theorem 2.8 .

The conditions of Lemma 3.5 and Theorem 3.6 seem difficult to check in practice. We now give an example where these results can be applied.

EXAmple. Let $\left\{\lambda_{i}\right\}_{i=1}^{m}$ be the eigenvalues of $B=I-A$ with the corre- 
sponding eigenvectors $\left\{\gamma_{i}\right\}_{i=1}^{m}$. Suppose that $\left|\lambda_{1}\right| \geq \ldots \geq\left|\lambda_{m}\right|$ and that the eigenvectors form a basis of $\mathbb{R}^{m}$. Let $r_{n}^{\prime}$ be such that $r_{n}^{\prime}=B r_{n-1}^{\prime}$ and let $r_{n}$ be obtained by the hybrid procedure from $r_{n}^{\prime}$ and $r_{n+1}^{\prime}$. Let $r_{0}^{\prime}=\sum_{i=1}^{m} d_{i} \gamma_{i}$. Thus

$$
r_{n}^{\prime}=\sum_{i=1}^{m} d_{i} \lambda_{i}^{n} \gamma_{i}=d_{1} \lambda_{1}^{n} \gamma_{1}+\sum_{i=2}^{m} d_{i} \lambda_{i}^{n} \gamma_{i} .
$$

Setting

$$
c_{n}=d_{1} \lambda_{1}^{n}, \quad a_{n}=\sum_{i=2}^{m} d_{i} \lambda_{i}^{n} \gamma_{i},
$$

we get from Remark 8 and Theorem 3.6

THEOREM 3.7. If $r_{n}^{\prime}=B r_{n-1}^{\prime}, r_{0}=r_{0}^{\prime}$ and if $r_{n}$ is obtained by the hybrid procedure from $r_{n}^{\prime}$ and $r_{n+1}^{\prime}$, then $\left\|r_{n}\right\|=\mathcal{O}\left(\left|\lambda_{2}\right|^{n}\right)$. Moreover, if $\left|\lambda_{2}\right|<\left|\lambda_{1}\right|$, then $\lim _{n \rightarrow \infty}\left\|r_{n}\right\| /\left\|r_{n}^{\prime}\right\|=0$.

Remark 9. This theorem holds even if $\left|\lambda_{2}\right|<1<\left|\lambda_{1}\right|$.

Remark 10. Since $\left(\alpha_{n}\right)$ converges, Lemma 3.4 shows that $\left(r_{n}\right)$ converges with the same speed as $\left(r_{n}^{\prime}\right)$. In this case, the iterations will be stopped when $\left|\alpha_{n}+\lambda_{1} /\left(1-\lambda_{1}\right)\right| \leq \varepsilon$, where $\varepsilon$ is an arbitrary threshold. Of course the value of $\lambda_{1}$ is usually unknown and this test cannot be used in practice. Thus the iterations will be stopped when $\left|\alpha_{n}-\alpha_{n-1}\right| \leq \varepsilon$. However, it must be noticed that, due to a possible stagnation of the method, this test does not guarantee that the recurrence is close to the limit.

4. Numerical examples. In all the examples we take $G=I, M=I$, $B=I-A$ and $x_{0}=0$. The right-hand side is computed in order that the solution be $\widetilde{x}=[1, \ldots, 1]^{T}$. Each figure shows $\log \left\|r_{n}^{\prime}\right\|$ and $\log \left\|r_{n}\right\|$ as a function of the number $n$ of iterations and the lowest curve always corresponds to the hybrid procedure.

Let $\left\{\lambda_{i}\right\}_{i=1}^{m}$ be the set of eigenvalues of $B$. The elements of the matrix $A \in \mathbb{R}^{100 \times 100}$ were randomly chosen in $[0,1]$. The values of $\|B\|,\left|\lambda_{i}\right|(i=$ $1, \ldots, 100)$ were computed with Matlab with a precision of $10^{-20}$.

4.1. Case (i). Let $r_{n}^{\prime}$ be obtained by the norm decomposition method of Gastinel [3] with $\varphi_{1}(r)=\sum_{i=1}^{m}\left|r_{i}\right|$. This method is as follows: for $n=0,1, \ldots$,

$$
x_{n+1}^{\prime}=x_{n}^{\prime}-\alpha_{n}^{\prime} A^{T} z_{n}, \quad r_{n+1}^{\prime}=r_{n}^{\prime}+\alpha_{n}^{\prime} A A^{T} z_{n},
$$

where $z_{n}=\operatorname{sgn}\left(r_{n}^{\prime}\right)$. Thus, $\left(z_{n}, r_{n}^{\prime}\right)=\varphi_{1}\left(r_{n}^{\prime}\right)$ and

$$
\alpha_{n}^{\prime}=-\frac{\varphi_{1}\left(r_{n}^{\prime}\right)}{\left(A^{T} z_{n}, A^{T} z_{n}\right)} .
$$


Let $r_{n}$ be computed by the hybrid procedure from $r_{n}^{\prime}$ and $B r_{n-1}$.

\begin{tabular}{cccc}
\hline & Example 1 & Example 2 & Example 3 \\
\hline$\|B\|$ & 0.998605 & 0.663839 & 1.485374 \\
$\left|\lambda_{1}\right|$ & 0.030418 & 0.661562 & 0.078104 \\
$\left|\lambda_{2}\right|$ & 0.030372 & 0.040093 & 0.046249 \\
\hline
\end{tabular}

For each example $\left|\lambda_{2}\right|<\left|\lambda_{1}\right|$ and thus condition 2 of Theorem 3.2 is satisfied. We did not check condition 1 but the numerical results show that, in this case, the convergence of Gastinel's method has been accelerated.
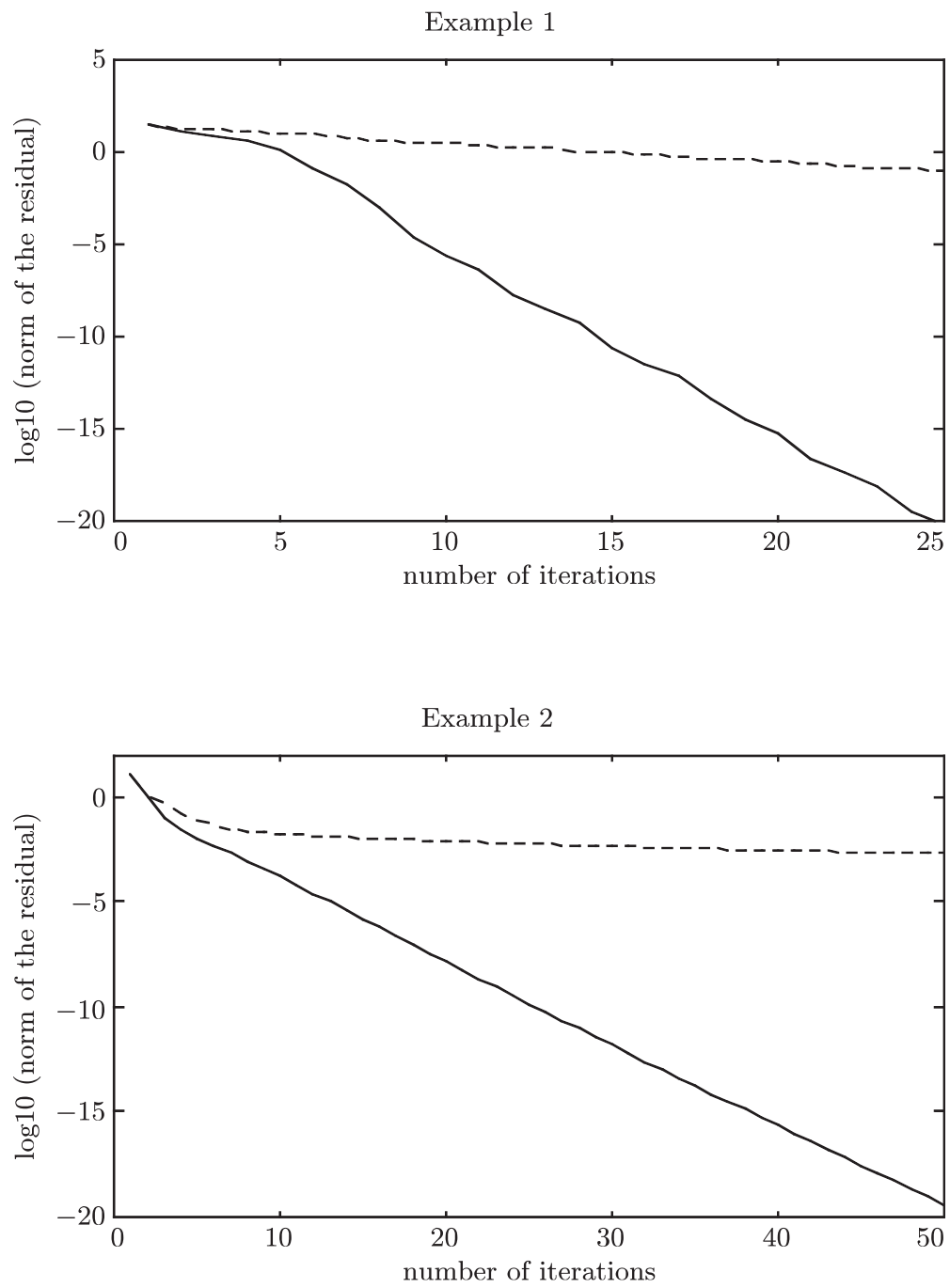


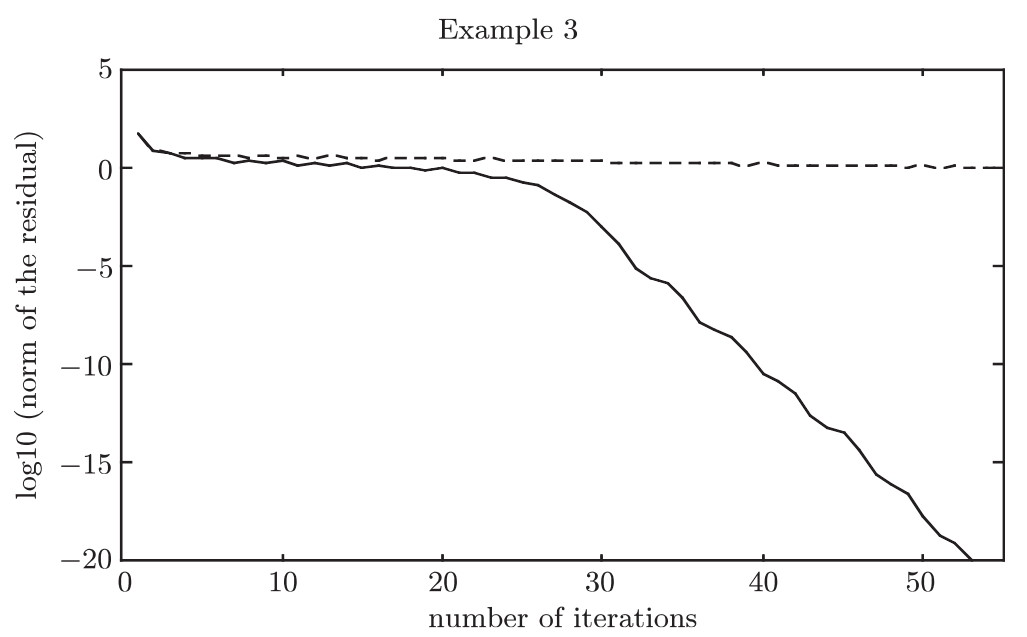

4.2. Case (ii). Let $r_{n}^{\prime}$ be such that $r_{n}^{\prime}=B r_{n-1}^{\prime}$ and let $r_{n}$ be computed by the hybrid procedure from $r_{n}^{\prime}$ and $r_{n+1}^{\prime}$.

\begin{tabular}{cccc}
\hline & Example 4 & Example 5 & Example 6 \\
\hline$\|B\|$ & 6.296298 & 3.273282 & 6.457731 \\
$\left|\lambda_{1}\right|$ & 6.274695 & 1.158723 & 0.822448 \\
$\left|\lambda_{2}\right|$ & 0.380272 & 0.099341 & 0.195185 \\
\hline
\end{tabular}

Let $N$ be the index such that $\left|\alpha_{N}+\lambda_{1} /\left(1-\lambda_{1}\right)\right| \leq 10^{-20}$. We get

\begin{tabular}{lccc}
\hline & Example 4 & Example 5 & Example 6 \\
\hline$N$ & 12 & $>35$ & $>55$ \\
$\log \left\|r_{N}^{\prime}\right\|$ & 20.992904 & & \\
$\log \left\|r_{N}\right\|$ & -9.771103 & & \\
\hline
\end{tabular}

Example 4

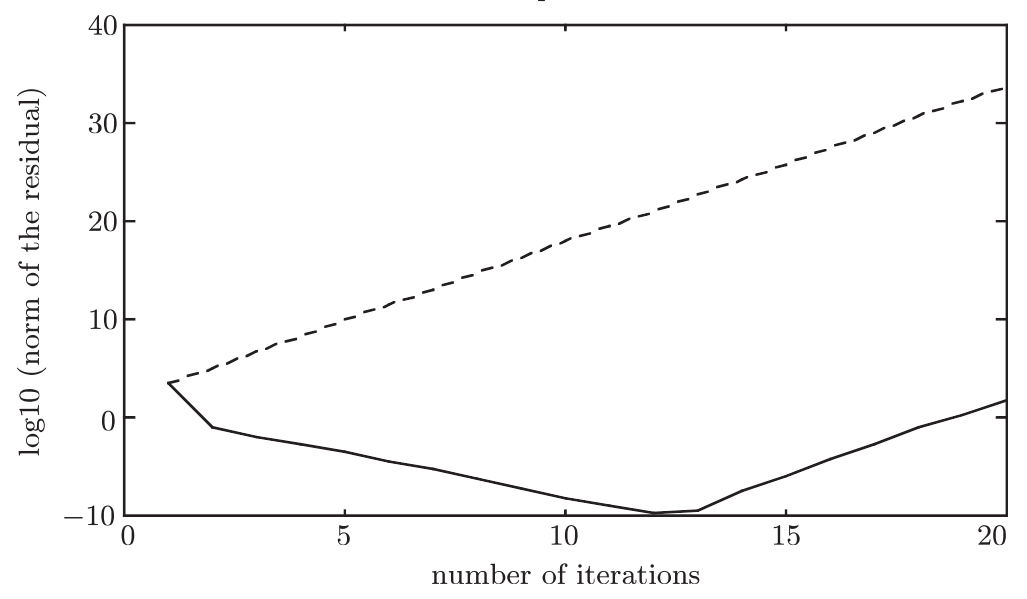



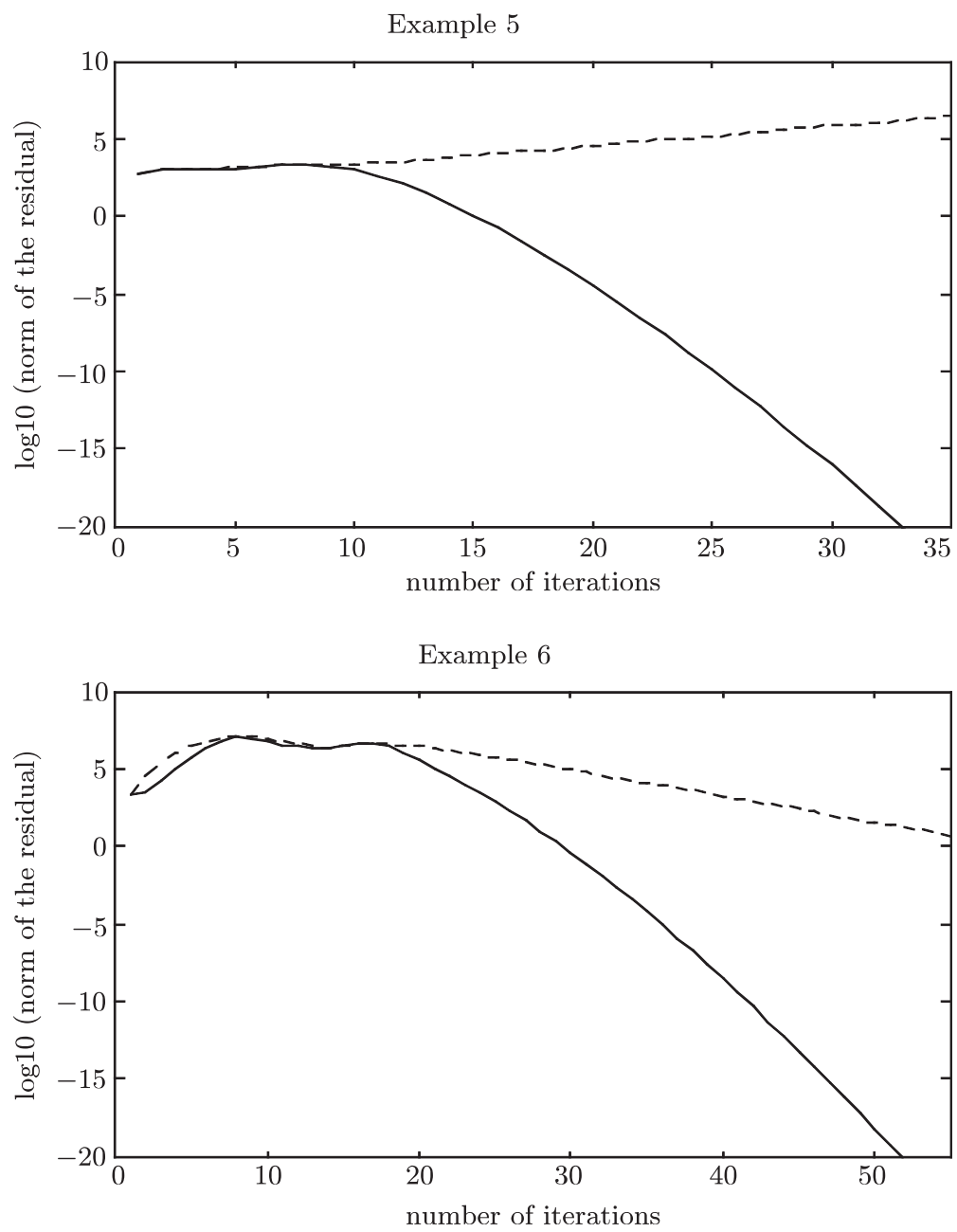

For each example we have $\left|\lambda_{2}\right|<\left|\lambda_{1}\right|$. Thus the conditions of Theorem 3.7 are satisfied and we get $\lim _{n \rightarrow \infty}\left\|r_{n}\right\| /\left\|r_{n}^{\prime}\right\|=0$ even if $\lim _{n \rightarrow \infty}\left\|r_{n}^{\prime}\right\|=\infty$ (see Examples 4 and 5). For Example 1 we get, at iteration 12, $\mid \alpha_{12}+$ $\lambda_{1} /\left(1-\lambda_{1}\right) \mid \leq 10^{-20}$. Moreover, we also have $\left|\alpha_{n}+\lambda_{1} /\left(1-\lambda_{1}\right)\right| \leq 10^{-20}$ for $n \in[12,20]$, and thus we see that $\left(r_{n}\right)$ converges with the same speed as $\left(r_{n}^{\prime}\right)$. We can also remark that, since the sequence $\left(r_{n}^{\prime}\right)$ diverges, so does $\left(r_{n}\right)$ (from iteration 12) and thus it is better to stop the iterations at $n=12$.

\section{References}

[1] C. Brezinski and M. Redivo Zaglia, Hybrid procedures for solving linear systems, Numer. Math. 67 (1994), 1-19. 
[2] R. W. Freund, A transpose-free quasi-minimal residual algorithm for non-Hermitian linear systems, SIAM J. Sci. Statist. Comput. 14 (1993), 470-482.

[3] N. Gastinel, Procédé itératif pour la résolution numérique d'un système d'équations linéaires, C. R. Acad. Sci. Paris 246 (1958), 2571-2574.

[4] K. Jbilou, Projection-minimization methods for nonsymmetric linear systems, Linear Algebra Appl. 229 (1995), 101-125.

[5] - G-orthogonal projection methods for solving linear systems, to appear.

[6] W. Schönauer, Scientific Computing on Vector Computers, North-Holland, Amsterdam, 1987.

[7] W. Schönauer, H. Müller and E. Schnepf, Numerical tests with biconjugate gradient type methods, Z. Angew. Math. Mech. 65 (1985), T400-T402.

[8] R. Weiss, Convergence behavior of generalized conjugate gradient methods, Ph.D. Thesis, University of Karlsruhe, 1990.

[9] —, Error-minimizing Krylov subspace methods, SIAM J. Sci. Statist. Comput. 15 (1994), 511-527.

[10] - Properties of generalized conjugate gradient methods, Numer. Linear Algebra Appl. 1 (1994), 45-63.

[11] R. Weiss and W. Schönauer, Accelerating generalized conjugate gradient methods by smoothing, in: Iterative Methods in Linear Algebra, R. Beauwens and P. de Groen (eds.), North-Holland, Amsterdam, 1992, 283-292.

[12] L. Zhou and H. F. Walker, Residual smoothing techniques for iterative methods, SIAM J. Sci. Statist. Comput. 15 (1994), 297-312.

Anna Abkowicz and Claude Brezinski

Laboratoire d'Analyse Numérique et d'Optimisation

UFR IEEA-M3

Université des Sciences et Technologies de Lille

F-59655 Villeneuve d'Ascq Cedex, France

E-mail: brezinsk@omega.univ-lille1.fr 